\title{
Características clínicas, predictores de mortalidad y resultados Inmediatos y a largo plazo en el tratamiento con angioplastía primaria del infarto agudo al miocardio con supradesnivel del ST complicado con shock cardiogénico
}

\author{
Martín Valdebenito, Nicolás Veas, Dante Lindefjeld, José Luis Winter, Eduardo Guarda, Osvaldo \\ Pérez, Manuel Méndez, Eugenio Marchant, Alejandro Fajuri, Alejandro Martínez. \\ División de Enfermedades Cardiovasculares, Centro de Terapia Endovascular, Pontificia \\ Universidad Católica. \\ Laboratorio de Hemodinamia, Hospital Dr. Sótero del Río.
}

Introducción: El shock cardiogénico (SC) es la complicación más grave del infarto agudo al miocardio (IAM). La mortalidad del SC varía entre un 40-60\%. Con el acceso a la angioplastía primaria, el pronóstico del SC ha mejorado en los últimos años, aunque la mortalidad permanece elevada. Nuestro objetivo fue analizar las características clínicas, predictores de mortalidad intrahospitalaria y a largo plazo de los pacientes con IAM complicados con SC en 3 centros hospitalarios con disponibilidad de hemodinamia de urgencia las 24 horas del día.

Métodos y Resultados. Estudio analítico observacional. Se incluyeron pacientes entre Enero 2009 y Agosto 2013. La mortalidad se confirmó por certificado de defunción emitido por el registro civil. En el período estudiado se encontraron 101 pacientes con IAM con SC, con una mortalidad intrahospitalaria de $40.8 \%$ y global de $53.5 \%$ con un seguimiento medio de 1.6 años. No hubo deiferencias significativas entre vivos y fallecidos, respecto de la edad de presentación, antecedentes $\mathrm{CV}$, enfermedad renal, PCR al momento de presentación, tiempo transcurrido desde la presentación del SC, trombo-aspiración, uso de balón de contrapulsación intraórtico (BCIAo), ni resultado angiográfico final. El grupo de mortalidad presentaba mayor número de vasos enfermos (83.3\% vs 63.2\%; p:0.012), peor fracción de eyección ( $\mathrm{Fe}<30 \% 66.7 \%$ vs $22.5 \%$; $\mathrm{p}<0,001)$. Los predictores ajustados de mortalidad intrahospitalaria fueron la presencia de 2 o más vasos enfermos OR 2.9 (IC $95 \%$ $1.19-7,1 ; \mathrm{p}=0.012$ ), y la FE < 30\% (OR 6.8, IC 95\% 2.23 - 21.27; $\mathrm{p}=0.001)$. Los predictores de mortalidad global fueron la $\mathrm{FE}<30 \%$ (HR 3.6, IC 95\% 1.4-9.2; $\mathrm{P}=0.02$ ) y la presencia de 2 o más vasos enfermos (HR 3.2 , IC 95\% 1.1-10.7). En 21 (16.5\%) se uso BCIAo. La mortalidad intrahospitalaria no difirió entre el grupo con BCIAo y el grupo de sólo tratamiento médico (47.6\% vs $38.75 \mathrm{P}=0.299$ ) como tampoco la mortalidad global (52.4\% vs $47.6 \%$ P: 0.579 ). Tampoco hubo diferencias en las curvas de sobrevida (log-rank $\mathrm{P}=0.693$ ). Sobre la mortalidad intrahospitalaria no encontramos diferencias significativas entre los grupos (31.5\% vs $44.9 \%$; p:0.091), ni tampoco en la mortalidad global (50\% vs $53,6 \%$; p:0.414) con una media de seguimiento de 1,6 años (0-4.5 años). No hubo diferencias en las curvas de sobrevida.

Conclusiones: El SC tiene alta mortalidad intrahospitalaria y global. En nuestra serie, los predictores de mortalidad fueron la disfunción sistólica severa y el número de vasos enfermos.

\section{Correspondencia:}

Dr. Martín Valdebenito.

Departamento de Enfermedades Cardiovasculares,

Pontificia Universidad Católica de Chile,

mvaldet@gmail.com 


\section{Clinical characteristics and predictors of early and global mortality in patients with ST segment elevation Myocardial Infarction and Cardiogenic Shock undergoing Primary Angioplasty}

Background. Cardiogenic shock in acute myocardial infarction (AMI) is associated with a high mortality rate $(40-60 \%)$. An improvement in this prognosis has followed the introduction of primary angioplasty. The aim of this study was to analyze the clinical and angiographic characteristics as well as to determine the early and late mortality in patients with St elevation AMI and cardiogenic shock undergoing primary angioplasty in 3 hospital centers where this procedure was available on a $24 \mathrm{hr}$ basis.

Methods and Results. We analyzed the clinical an angiographic data of patients treated with primary PTCA for AMI and cardiogenic shock from January 2009 to August 2013. Mortality was confirmed by the data from a National Statistics Office. 101 patients were included and followed for an average of 1.6 years after primary PTCA. Early and total mortality rates were $40.8 \%$ and $53.5 \%$, respectively. No differences were found between survivors and non survivors regarding age, risk factors, previous cardiovascular disease, renal failure, CRP and time to PTCA. Intracoronary thrombus aspiration, use of intra-aortic balloon pumping or final angiographic findings were also similar between groups. Compared to survivors, deceased patients had more vessels involved ( $>=2$ vessels in $83.3 \%$ vs $63.2 \%$, respectively, $\mathrm{p}=0.012$ ) and lower EF (under $30 \%$ in $66.7 \%$ vs $22.5 \%$, respectively, $\mathrm{p}<0.001)$. Predictors of hospital mortality were the presence of $>=2$ vessel disease (OR 2.9, 95\% C.I. $1.19-7.1, \mathrm{p}=0.012$ ) and $\mathrm{EF}$ under $30 \%$ (OR 6.8, C.I. $2.23-21.27, \mathrm{p}=0.001)$. Global mortality predictors were $\mathrm{EF}<30 \%$ (HR 3.6, IC 95\% 1.4-9.2; $\mathrm{P}=0.02$ ) and the presence of $>=2$ vessel disease (HR 3.2, IC $95 \%$ 1.1-10.7). No difference in hospital mortality was observed between patients undergoing intra-aortic balloon pumping (16.5\%) as opposed to those without such support $(47.6 \%$ vs $38.75 \mathrm{P}=0.299$, respectively). Similar absence of effect from IABP applied to global mortality. Survival curves up to 1.6 years of follow-up were also similar in both groups.

Conclusion. Cardiogenic shock in AMI leads to a high hospital and global mortality. Significant predictors of mortality were severe left ventricular dysfunction and multivessel disease

Key words: myocardial infarction, cardiogenic shock, primary angioplasty, mortality . 


\section{Introducción}

El shock cardiogénico (SC) asociado al ianfarto al miocardio lleva a un estado de hipoperfusión generalizada con falla macro y microhemodinámica ${ }^{1}$. Sin intervención efectiva, la progresión del shock es rápida y fatal.

En Estados Unidos y en Europa ocurren al año aproximadamente unos 500 mil y 650 mil infartos agudos al miocardio con elevación del ST (IAM c/SDST), respectivamente $^{7}$. La incidencia de SC en paciente hospitalizados por IAM c/SDST es de 5 a $10 \%$ y de 2-3 \% en IAM sin supradesnivel del ST (IAM s/SDST). En Chile, en el registro GEMI se han constatado 7548 IAM c/SDST entre el año 2001 al 2009, de los cuales un 3,7\% desarrolló un SC. Se observó una mortalidad global de $55 \%$, siendo significativamente menor la diferencia en hospitales con capacidad de angioplastía las 24 horas en comparados con aquellos sin disponibilidad de ésta ( $49 \%$ vs $62 \%){ }^{8}$

El estudio SHOCK ${ }^{2}$ demostró que pacientes con Infarto Agudo al Miocardio (IAM) complicado por SC obtenían un beneficio en mortalidad a 30 días si se lograba una revascularización precoz en comparación a la estabilización y tratamiento médico ( $46.7 \%$ vs $56 \%$ ).

El objetivo de nuestro estudio fue analizar las características clínicas de presentación, predictores de mortalidad, seguimiento intrahospitalario y a largo plazo de los pacientes con IAM complicados con SC que se presentaron en 3 centros hospitalarios de alta complejidad con disponibilidad de laboratorio de hemodinamia de urgencia las 24 horas del día.

\section{Métodos}

\section{Pacientes}

Se incluyeron todos los pacientes con IAM c/SDST complicados con SC tratados con angioplastía primaria en el Hospital Clínico de la Universidad Católica, Clínica UC San Carlos de Apoquindo y en el Hospital Dr. Sótero del Río entre Enero 2009 y Agosto 2013.

Los criterios de inclusión fueron pacientes $>18$ años, que se presentaron con IAM c/ SDST complicados con SC sometidos a angioplastía primaria. Los criterios de exclusión fueron enfermedad sistémica severa, imposibilidad de revascularización y antecedente de cirugía o trombolisis reciente. El diseño fue analítico observacional. Los datos se recolectaron de la revisión de registros en fichas electrónicas y del protocolo del procedimiento efectuado. $\mathrm{La}$ mortalidad alejada se estableció a través del certificado de defunción emitido por el registro civil.

\section{Angioplastía Primaria}

Se consideró una angioplastía primaria al procedimiento realizado en pacientes con angina $>30$ minutos y presencia de SDST $>1 \mathrm{~mm}$, en al menos dos derivaciones contiguas. Previo a la PTCA los pacientes con IAM c/SDST, recibieron en el servicio de urgencia aspirina, betabloqueadores, nitroglicerina, heparina o analgésicos. Durante la intervención se mantuvo un tiempo de coagulación (ACT) $>300$ segundos y se administró $600 \mathrm{mg}$ de clopidogrel. El uso de inhibidores IIb/IIIa, nitroglicerina, adenosina, verapamilo, nitroprusiato, predilatación o postdilatación con balón quedó a discreción del operador.

\section{Definiciones}

a) IAM: Se definió como IAM la presencia de dolor torácico asociado a elevación del segmento ST, una onda Q patológica, un nuevo bloqueo de rama izquierda o una localización posterior del infarto con depresión del segmento ST anterior y alza de biomarcadores (troponinas, CK Total y CK MB).

b) Shock Cardiogénico: Presencia de presión arterial sistólica $<90 \mathrm{mmHg}$ por más de 30 minutos, que no responde con aporte de volumen y se mantiene una vez corregido los trastornos del ritmo, asociado a la presencia de signos de hipoperfusión (piel fría y sudorosa, oliguria o estado mental alterado).

c) Mortalidad Intrahospitalaria y Global: se consideró mortalidad intrahospitalaria la ocurrida durante la estadía hospitalaria y mortalidad global la ocurrida desde el momento del IAM hasta el fin del período de seguimiento.

\section{Análisis Estadístico}

Se usaron estadísticas descriptivas tales como media, desviación estándar y frecuencia, que fueron calculadas para cada característica clínica y demográfica si era apropiada. Además se utilizaron pruebas estadísticas de chi-cuadrado, test de mann-whitney, t-student según correspondiera. Se realizó análisis univariable, regresión logística múltiple y cálculo de Odds Ratio (OR) para mortalidad intrahospitalaria y Hazard Ratio (HR) para la alejada, además de curvas de sobrevida y test de log-rank.

\section{Resultados}

\section{1- Características de los pacientes}

En el período estudiado (4 años y medio) se identificaron 1886 pacientes con IAM c/SDST, de los cuales 101 presen- 
taron SC (5.35\%). Se apreció una distribución de factores de riesgo habitual para IAM, pero destacó la elevada tasa de paro cardiorespiratorio como forma de presentación. En algo menos de la mitad de los pacientes se encontró enfermedad de 3 vasos, en un cuarto de los pacientes enfermedad de 2 vasos y en otro cuarto enfermedad de 1 vaso. Solo 8 pacientes $(6,3 \%)$ tenían enfermedad de Tronco Coronario Izquierdo (TCI) +3 vasos. (Tabla 1)

La arteria descendente anterior (DA) fue la arteria más frecuentemente responsable del IAM $(45,6 \%)$, seguida de la arteria coronaria derecha (CD) (34\%). Hubo compromiso de TCI en $8,6 \%$ de los pacientes y de arteria circunfleja (ACx) en $11.8 \%$ de los pacientes. Se midió la fracción de eyección por ventriculografía en $75 \%$ del total de pacientes: $57 \%$ de ellos enían $\mathrm{FE}<30 \%$ y $43 \%$ entre $30-50 \%$. (Tabla 1)

Tabla 1. Características Clínicas y angiográficas de pacientes con IAM y Shock cardiogénico (N: 101)

$\begin{array}{lr}\text { Edad (promedio) } & 61,7 \text { años (38-87) } \\ \text { Sexo Masculino } & 59 \%(75) \\ \text { HTA } & 59 \%(75) \\ \text { Diabetes Mellitus } 2 & 43,3 \%(54) \\ \text { Dislipidemia } & 14,1 \%(18) \\ \text { Tabaquismo } & 26,7 \%(34) \\ \text { Usuario de Cocaína } & 3,14 \%(4) \\ \text { IAM previo } & 5,5 \%(7) \\ \text { Cirugía de Revascularización Previa } & 2,4 \%(3) \\ \text { Presentación de SC <24 hrs del IAM } & 76 \%(96) \\ \text { Paro Cardio- respiratorio en presentación } & 20,4 \%(26) \\ \text { Número de Vasos Enfermo } & \\ 3 \text { vasos } & 41,7 \%(53) \\ 2 \text { vasos } & 26 \%(33) \\ 1 \text { vaso } & 26 \%(33) \\ \text { TCl + 3 vasos } & 6,3 \%(8) \\ \text { Arteria Responsable del IAM } & \\ \text { TCl } & 8,6 \%(11) \\ \text { DA } & 45,6 \%(58) \\ \text { ACx } & 11,8 \%(15) \\ \text { ACD } & 34 \%(43) \\ \text { Fracción de Eyección } & \\ <30 \% & 57 \% \\ 30-50 & 43 \% \\ & \end{array}$

\section{2- Características del Procedimiento:}

La mayoría de los pacientes (algo menos de 2/3) recibió 1 stent; 2 stents recibieron aproximadamente $1 / 3$ y sólo $5 \%$ recibieron 3 o 4 . El promedio fue 1,5 stents por paciente. En algo menos de 1/5 de los pacientes se realizó aspiración de trombos intracoronarios con catéter. Muy pocos pacientes presentaron fenómeno de no-reflow como complicación de la angioplastía. El flujo TIMI 3 se obtuvo en $86 \%$ de las angioplastías y la revascularización fue completa en $80 \%$ de los casos (Tabla 2)

Tabla 2. Características del Procedimiento de Angioplastía Primaria en 101 pacientes con IAM y SDST con Shock cardiogénico

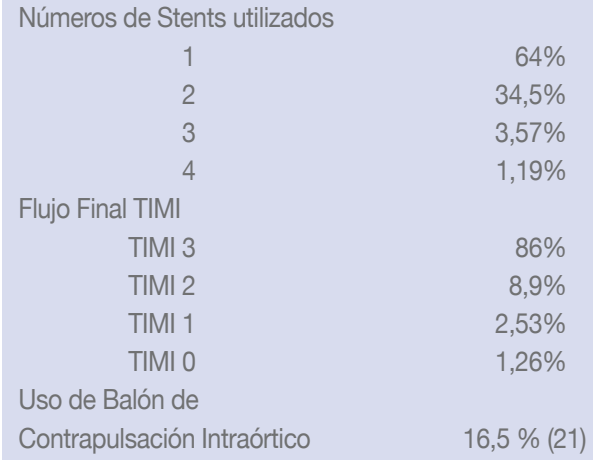

El balón de contrapulsación intra aórtica se usó en 16\% de los casos. Los pacientes con BCIA tenían menos HTA ( $38 \%$ vs $63 \%$; $\mathrm{P}=0.02$ ), más tabaquismo ( $47 \%$ vs $22 \%$, $\mathrm{P}=0.02)$, peor $\mathrm{FE}(<30 \%$ en $61 \%$ vs $27 \% ; \mathrm{P}=0.01)$ y mayor frecuencia de aspiración de trombos intracoronarios ( $38 \%$ vs $17 \%$; $\mathrm{P}=0.04)$. No hubo diferencias estadísticamente significativas entre el tiempo de presentación y estudio coronario, número de vasos enfermos, número de vasos tratados y resultado angiográfico final entre pacientes con o sin uso de BCIA (Tabla 2).

\section{3- Mortalidad:}

La mortalidad intrahospitalaria fue $40.8 \%$ y la global $53.5 \%$ con un seguimiento medio de 1,6 años ( 3 a 59 meses). Al comparar las características basales entre vivos y muertos no encontramos diferencias significativas entre la edad de presentación, antecedentes CV, PCR al momento de presentación, tiempo transcurrido entre presentación del SC y angioplastía primaria, trombo-aspiración, ni resultado angiográfico final. En cambio, el grupo con mayor mortalidad presentaba mayor número de vasos enfermos y peor fracción de eyección. (Tablas 3 y 4).

Los predictores ajustados de mortalidad intrahospitalaria fueron la presencia de 2 o más vasos enfermos y la FE $<30 \%$. (Tabla 3). Predictores de mortalidad global fueron la $\mathrm{FE}<30 \%$ y la presencia de 2 o más vasos enfermos (Tabla 4). 


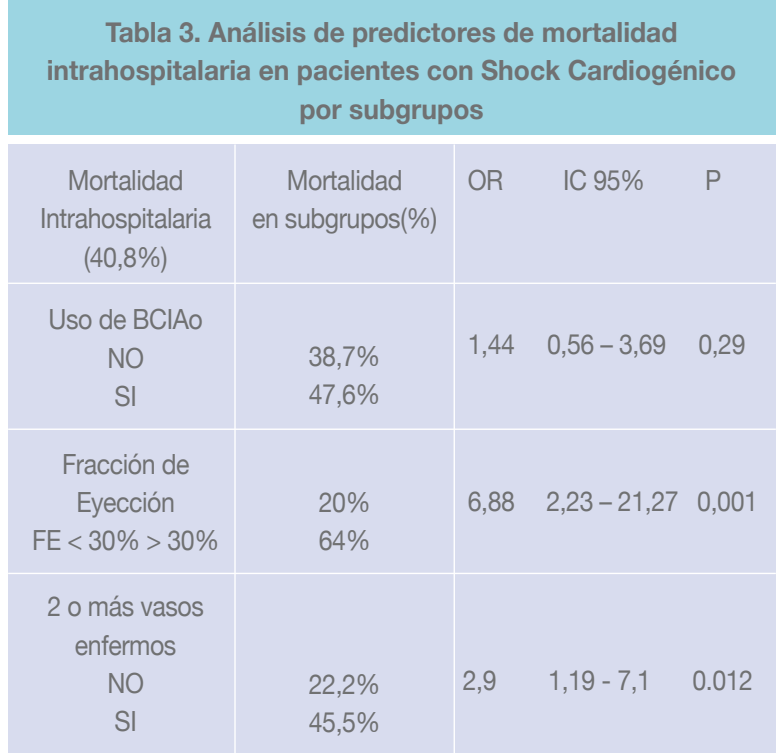

BCIAo: Balón de contrapulsación intraórtico. OR: Odds Ratio

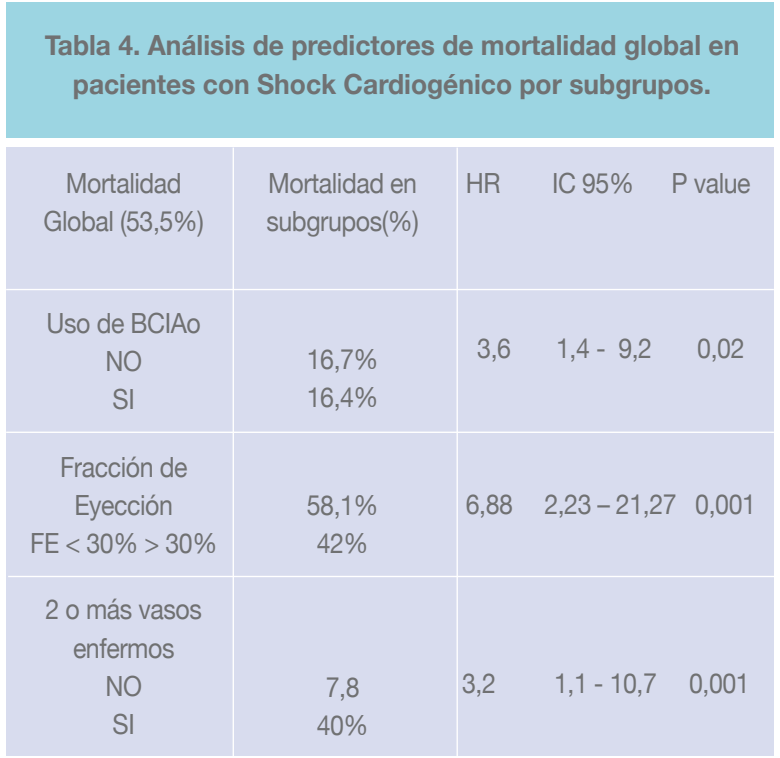

BCIAo: Balón de contrapulsación intraórtico. HR: Hazard Ratio.

No hubo diferencias significativas entre los pacientes tratados con BCIAo vs lo que no lo fueron respecto de la mortalidad intrahospitalaria ( $47.6 \%$ vs $38.75 \mathrm{P}=0.299$ ) ni de la mortalidad global (52.4\% vs $47.6 \%$ P: 0.579 ), con seguimiento medio de 1.6 años (Hazard Ratio (HR) con BCIAo de 0.98 , IC 95\% 0.38-2.5, $\mathrm{P}=0.579)$. No hubo diferencias en las curvas de sobrevida (log-rank $\mathrm{P}=0.693$ ). En este estudio evaluamos las características clínicas, predictores de mortalidad y seguimiento a corto y largo plazo de los pacientes con un IAM complicado con shock car- diogénico tratados con angioplastía primaria. Nos encontramos que un $5.7 \%$ de los pacientes con IAM c/SDST se complicaron con un shock cardiogénico, lo cual se ajusta a reportes descriptivos previos ${ }^{1-7}$. La distribución de factores de riesgo en los pacientes con SC fue similar a la reportada en pacientes con IAM sin SC. La mayor proporción de pacientes tenía una disfunción sistólica severa y tenían enfermedad multivaso, siendo la arteria descendente anterior la más frecuentemente afectada. La mortalidad intrahospitalaria y global $(40,8 \%$ y $53,5 \%$, respectivamente) es similar a reportes internacionales actuales ${ }^{3}$, y se asemeja a lo reportado en el grupo GEMI ${ }^{8}$ para hospitales con disponibilidad de angioplastía las 24 horas del día.

Un $43 \%$ de los pacientes en quienes se realizó una ventriculografía tenían una FE de 30-50\% y no una disfunción sistólica severa $(<30 \%)$ característica del SC. Esto podría ser explicado por la injuria aguda que significa un IAM c/ SDST, situación en que los mecanismos compensatorios propios de una insuficiencia cardíaca crónica no alcanzan a estabilizar los síntomas y signos, llevando al SC. Se ha reportado que la mitad de los pacientes en shock cardiogénico tienen una FE de $30 \%{ }^{1-9}$, lo que en el contexto de isquemia miocárdica crónica produciría síntomas leves a moderados.

En un $86 \%$ de los casos el procedimiento se consideró exitoso desde el punto de vista angiográfico, con flujo final TIMI 3. El balón de contrapulsación se instaló en un $20,9 \%$ de los pacientes. La utilidad de la implantación de BCIAo ha sido motivo de debate, en diversos estudios, especialmente en el SCHOK II. ${ }^{10-11}$ En nuestra serie encontramos que no hubo una mejoría de la mortalidad, dato que, junto a observaciones de literatura reciente, cuestionan la utilidad de un BCIAo en el SC.

Los predictores de mortalidad encontrados en nuestra serie fueron la $\mathrm{FE}<30 \%$ en la ventriculografía en el momento de la angioplastía y la presencia de enfermedad de 2 o más vasos.

Se han hecho intentos de definir marcadores clínicos objetivos que predicen muerte en pacientes con shock cardiogénico. Den Uil et al, mostró que las mediciones del daño de la microcirculación usando imágenes en campo oscuro (MicroScan $^{\mathrm{TM}}$, Microvision Medical, Amsterdam, Netherlands) podría predecir resultados adversos en pacientes con shock cardiogénico, un elemento a considerar en futuras búsqueda de nuevos predictores de riesgo ${ }^{12}$.

Estos datos sugieren que en presencia de predictores de mayor mortalidad el pronóstico de estos pacientes podría mejorar con un manejo más agresivo. Ello incluye, además de la angioplastía primaria multivaso, un monitoreo 
hemodinámico invasivo y acceso a dispositivos de asistencia ventricular como puente a la recuperación, destinación o trasplante cardíaco. La evidencia para el uso de estos dispositivos en pacientes con SC es derivada de trabajos observacionales, donde un número sustancial de pacientes ha tenido resultados favorables. Sin embargo, actualmente, muy pocos pacientes tienen acceso a este tipo de soporte avanzado, incluso después de una intervención coronaria precoz y efectiva. ${ }^{13-14-15-16}$

Nuestro trabajo tiene algunas limitaciones. Se trata de un estudio retrospectivo y la definición utilizada para el SC es clínica, sin emplear una definición hemodinámica globalmente aceptada. Además, no obtuvimos las mediciones hemodinámicas o marcadores inflamatorios en el período intrahospitalario, como ha sido el caso en estudios previos $^{17-20}$. Por otra parte, al tratarse de una muestra de pacientes de tres centros con disponibilidad de servicio de hemodinamia las 24 horas del día y de unidad de cuidados intensivos con experiencia en el manejo de pacientes críticos coronarios, es difícil plantear que los resultados puedan extrapolarse a la realidad nacional.

\section{Conclusión}

El shock cardiogénico es una complicación del IAM que sigue teniendo una alta tasa de mortalidad intrahospitalaria y global a pesar de un acceso a reperfusión precoz y medidas de soporte avanzadas en unidades de cuidados intensivos. Los predictores importantes de mortalidad fueron la enfermedad de 2 o más vasos y la $\mathrm{FE}<30 \%$. El balón de contrapulsación intraaórtico, al igual que en otras series, no mostró mejorar la sobrevida de los pacientes

Conflicto de Intereses: Ninguno de los autores declara conflictos de intereses en relación a la información presentada.

\section{Referencias:}

1- HASDAI D1, TOPOL EJ, CALIFF RM, BERGER PB, HOLMES DR JR. Cardiogenic Shock Complicating Acute Coronary Syndromes. Lancet 2000; 356, 749-756

2- HOCHMAN JS, SLEEPER LA, WEBB JG, SANBORN TA, WHITE HD, TALLEY JD, et al. Early Revascularization in acute myocardial infarction complicated by cardiogenic shock. N engl J Med 1999; 341: 625.

3- BABAEV A1, FREDERICK PD, PASTA DJ, EVERY N, SICHROVSKY T, HOCHMAN JS,et al. Trends in management and outcomes of patients with acute myocardial infarction complicated by cardiogenic shock. JAMA 2005; 294:448-454.

4- GOLDBERG RJ, SPENCER F, GORE J, LESSARD D, YARZEBSKI K. Thirty-year trends (1975-2005) in the magnitude of, management of, and hospital death rates associated with cardiogenic shock in patients with acute myocardial infarction: a population-based perspective. Circulation 2009; 119: 12111219.

5- JEGER RV, RADOVANOVIC D, HUNZIKER PR, PFISTERER ME, STAUFFER JC, ERNE P, et al. Ten-year trends in the incidence and treatment of cardiogenic shock. Ann. Intern. Med.2008; 149: 618-626.

6- FANG J, MENSAH GA, ALDERMAN MH, CROFT JB. Trends in acute myocardial infarction complicated by cardiogenic shock 1979-2003, United States. Am. Heart J. 2006; 152: 1035-1041.

7- THOM T, HAASE N, ROSAMOND W, HOWARD VJ, 
RUMSFELD J, MANOLIO T, et al. Heart disease and stroke statistics: a report from the american Heart Association Statistics Committee and Stroke Statistics Committee. Circulation 2006; 113: e85-e151.

8- SEPÚLVEDA P, NAZZAL C, SOTO G, PRIETO JC, SANHUEZA P, CAMPOS P, et al. Características clínicas y tratamiento de pacientes con infarto agudo del miocardio con supradesnivel del ST y shock cardiogénico: 9 años del Registro GEMI. Rev Chil Cardiol 2012; 31: $194-201$.

9- HOCHMAN JS, BOLAND J, SLEEPER LA, PORWAY M, BRINKER J, COL J, et al. Current spectrum of cardiogenic shock and effect of early revascularization on mortality. Results of an International Registry. SHOCK Registry Investigators. Circulation 1995; 91: 873-881.

10- THIELE H, ZEYMER U, NEUMANN FJ, FERENC M, OLBRICH HG, HAUSLEITER J, et al. Intraaortic Balloon Support for Myocardial Infarction with Cardiogenic Shock. N Engl J Med $2012 ; 367: 1287-96$

11- SJAUW KD, ENGSTRÖM AE, VIS MM, VAN DER SCHAAF RJ, BAAN J JR, KOCH KT, et al. A systematic review and meta-analysis of intra-aortic balloon pump therapy in ST elevation myocardial infarction: should we change the guidelines? Eur Heart J 2009;30:459-68.

12- DEN UIL CA, LAGRAND WK, VAN DER ENT M, JEWBALI LS, CHENG JM, SPRONK PE, et al. Impaired microcirculation predicts por outcome of patients with acute myocrdial infarction complicated by cardiogenic shock. Eur Heart J, 2010; 31: 3032-9.

13- THIELE H, ALLAM B, CHATELLIER G, SCHULER G, LAFONT A. A Shock in acute myocardial infarction: The Cape
Horn for trials?. Eur Heart J 2010; 31: 1828-35.

14- ARZAMENDI D1, GARCIA-ALVAREZ A, ROIG E, BENITO B, KIAMCO R, PEREZ-VILLA F, et al. New Insights into the management of cardiogenic shock complicating myocardial infarction: role of urgent heart transplantation. J Heart Lung Transplant 2008; 27: 984-9.

15- KRISHNAMANI R1, DENOFRIO D, KONSTAM MA. Emerging ventricular assit devices for long-term cardiac support. Nat Rev Cardiol 2010; 7: 71-76.

16- REYNOLDS HR1, HOCHMAN JS. Cardiogenic shock: current concepts and improving outcomes. Circulation 2008; 117: 686-697.

17- THIELE H, LAUER B, HAMBRECHT R, BOUDRIOT E, COHEN HA, SCHULER G. Reversal of cardiogenic shock by percutaneous leftatrial- to-femoral arterial bypass assistance. Circulation 2001; 104: 2917-22.

18- THIELE H, LAUER B, HAMBRECHT R, et al. Randomized comparison of intra-aortic balloon support versus a percutaneous left ventricular assist device in patients with revascularized acute myocardial infarction complicated by cardiogenic shock. Eur Heart J 2005; 26:1276-83.

19- FINCKE R, HOCHMAN JS, LOWE AM, MENON V, SLATER JN, WEBB JG, et al. Cardiac power is the strongest hemodynamic correlate of mortality in cardiogenic shock: a report from the SHOCK trial registry. J Am Coll Cardiol 2004; 44:340-8.

20- PRONDZINSKY R1, UNVERZAGT S, LEMM H, WEGENER NA, SCHLITT A, HEINROTH KM, et al. Interleukin-6, $-7,-8$ and -10 predict outcome in acute myocardial infarction complicated by cardiogenic shock. Clin Res Cardiol 2012; 101: 375-84. 